\title{
Using linear polarization for sensing and monitoring nanoparticle purity
}

\author{
Ángela I. Barreda ${ }^{\mathrm{a}}$, Juan M. Sanz ${ }^{\mathrm{a}}$, Rodrigo Alcaraz de la Osa ${ }^{\mathrm{a}}$, José M. Saiz ${ }^{\mathrm{a}}$, Fernando Moreno ${ }^{\mathrm{a}}$, \\ Francisco González ${ }^{\mathrm{a}}$, Gorden Videen ${ }^{\mathrm{a}, \mathrm{b}, \mathrm{c}}$
}

${ }^{a}$ Grupo de Óptica, Departamento de Física Aplicada, Universidad de Cantabria, Facultad de Ciencias, Avda. Los Castros s/n, 39005 Santander, Spain; ' Instituto Nacional de Técnica Aerospacial (INTA), Ctra. Ajalvir Km. 4, 28850 Torrejón de Ardoz, Madrid, Spain;Army Research Laboratory, AMSRL-CI-EM, 2800 Powder Mill Road, Adelphi, 20783 MD, USA

\begin{abstract}
We analyze the effect of contaminants on the quadrupolar magnetic, dipolar electric and dipolar magnetic resonances of silicon nanoparticles (NPs) by considering the spectral evolution of the linear polarization degree at right angle scattering configuration, $\mathrm{P}_{\mathrm{L}}\left(90^{\circ}\right)$. From an optical point of view, a decrease in the purity of silicon nanoparticles due to the presence of contaminants impacts the NP effective refractive index. We study this effect for a silicon nanosphere of radius $200 \mathrm{~nm}$ embedded in different media. The weakness of the resonances induced on the $\mathrm{P}_{\mathrm{L}}\left(90^{\circ}\right)$ spectrum because of the lack of purity can be used to quantify the contamination of the material. In addition, it is shown that Kerker conditions also suffer from a spectral shift, which is quantified as a function of material purity.
\end{abstract}

Keywords: Nanoparticles, resonances, polarimetry, sensing, contaminants

\section{INTRODUCTION}

Nanotechnology has revolutionized science over the last several years, generating important theoretical and practical developments. The interaction of light with metallic NPs has undergone vigorous investigation providing many different applications like new sources and detectors ${ }^{1}$. When incident light illuminates a metallic NP, free electrons oscillate with the same frequency as the incident radiation, generating Localized Surface Plasmons (LSPs). These coherent oscillations of the electronic plasma depend on the optical properties of the NP, its size, shape and the wavelength of the incident radiation $^{2}$. At certain frequencies, resonances are produced. In these cases, the energy of the impinging radiation is transferred to free electrons, which oscillate at maximum amplitude, and strong enhancements of the electric field are observed in the NP surroundings. In spite of the strong response of metallic NPs in infrared and visible ranges, they are useless in many practical applications due to their inherent ohmic losses. High Refractive Index (HRI) dielectric NPs have been proposed as a solution to this drawback ${ }^{3}$. Some of the most interesting properties of this kind of NPs are that light can travel through them without being absorbed and that they present magnetic effects even for non-magnetic $(\mu=1)$ $\mathrm{NPs}^{4-6}$. This magneto-dielectric behavior is responsible for important directionality properties. Under certain conditions of the electric permittivity $\varepsilon$ and magnetic permeability $\mu$, proposed by Kerker et $a^{7,8}$, the forward and backward scattered intensity is almost null or null respectively. We will call those conditions as Scattering Directionality Conditions (SDCs). The resonances depend on the size, shape and optical properties of the NPs. For a given material, resonances are red-shifted as NP size increases, and they also are influenced by the refractive index of the surrounding medium, $\mathrm{m}_{\text {med }}$. Particularly, for HRI dielectric materials, resonances are red-shifted as $\mathrm{m}_{\text {med }}$ increases, in addition to being strengthened ${ }^{9}$.

From an industrial point of view, it is really complex to obtain $100 \%$ purity grade, with the corresponding increased price. In this sense, the effect of the purity of a silicon (Si) NP on the light-scattering extinction spectrum was analyzed ${ }^{10}$. In recent research, it was found that the magnetic resonances are more sensitive to changes in the purity than the electric resonances, and as the purity decreases, the magnetic resonances weakened. This behavior can be explained as the Joule's losses are much greater for the magnetic resonances than those for the electric ones. As it was expected, the

Optical Sensing and Detection IV, edited by Francis Berghmans, Anna G. Mignani, Proc. of SPIE Vol. 9899, 989910 - (C) 2016 SPIE · CCC code: 0277-786X/16/\$18 · doi: 10.1117/12.2227774 
SDCs are influenced by the presence of contaminants. In particular, the near Zero-Forward condition is shifted to longer wavelengths as the purity decreases. However, the Zero-Backward condition is blue-shifted as purity decreases.

The linear polarization degree of light scattered perpendicular to the incident beam, $\mathrm{P}_{\mathrm{L}}\left(90^{\circ}\right)$, is a polarimetric parameter that contains information about the magnetic or electric response of the resonances ${ }^{11,12}$. It can be used for determining the NP size and $\mathrm{m}_{\text {med }}$, which makes it a promising tool for sensing applications ${ }^{13}$.

In this work, using the Lorenz-Mie theory and focusing on $b_{2}, a_{1}$ and $b_{1}$ (being $b_{2}, a_{1}$ and $b_{1}$ the scattering coefficients of the quadrupolar magnetic, dipolar electric and dipolar magnetic resonances respectively), we analyze the dependence of $\mathrm{P}_{\mathrm{L}}\left(90^{\circ}\right)$ on the purity of spherical Si NPs embedded in different media with refractive indices $\mathrm{m}_{\text {med }}$ in the range $[1,1.5]$, (monitoring NPs purity for different $\mathrm{m}_{\mathrm{med}}$ ). On the other hand, the spectral shift of the Kerker conditions induced in the $\mathrm{P}_{\mathrm{L}}\left(90^{\circ}\right)$ values due to changes in the purity of the NPs also is analyzed. Finally, we study the changes in $\mathrm{P}_{\mathrm{L}}\left(90^{\circ}\right)$ due to variations in $\mathrm{m}_{\mathrm{med}}$ for different purity grades of the NP material, (sensing to $\mathrm{m}_{\mathrm{med}}$ for different purity grades).

The work is organized as follows: in Section 2, we introduce the theoretical background. In Section 3, we show the main results of this research. In Section 3.1 we study the changes $\mathrm{P}_{\mathrm{L}}\left(90^{\circ}\right)$ as a function of NP purity for different surrounding

refractive indices $\mathrm{m}_{\mathrm{med}} \in[1,1.5]$. In Section 3.2 we analyze the spectral shift exhibited in the $\mathrm{P}_{\mathrm{L}}\left(90^{\circ}\right)$ for Kerker conditions. In Section 3.3 we explore the influence of the NP purity on the behavior of $\mathrm{P}_{\mathrm{L}}\left(90^{\circ}\right)$ as a function of $\mathrm{m}_{\text {med. }}$. In Section 4 , we present the conclusions.

\section{THEORETICAL METHODS}

According to the Lorenz-Mie theory ${ }^{14}$, the extinction and scattering cross-sections by a spherical particle are given by the following expressions:

$$
\begin{aligned}
& C_{\text {ext }}=\frac{2 \cdot \pi}{k^{2}} \sum_{n=1}^{\infty}(2 \cdot n+1) \operatorname{Re}\left\{a_{n}+b_{n}\right\} \\
& C_{s c a}=\frac{2 \cdot \pi}{k^{2}} \sum_{n=1}^{\infty}(2 \cdot n+1)\left(\left|a_{n}\right|^{2}+\left|b_{n}\right|^{2}\right)
\end{aligned}
$$

where $a_{n}$ and $b_{n}$ are the well-known scattering coefficients of the Lorenz-Mie theory, both of which depend on the particle size and on the electric and magnetic properties of the NP relative to its surrounding medium. The wavenumber $\mathrm{k}$ is defined as $\left(2 \pi \mathrm{m}_{\mathrm{med}}\right) / \lambda$, where $\lambda$ is the wavelength of incident light in vacuum. The scattering coefficients $\mathrm{a}_{\mathrm{n}}$ and $\mathrm{b}_{\mathrm{n}}$ are the weights that appear in the linear expansion of vector spherical harmonics and indicate the strength of the multipolar contributions of order $n$. In particular, $a_{1}$ and $b_{1}$ correspond to the electric and magnetic dipolar modes, respectively, and $\mathrm{a}_{2}$ and $\mathrm{b}_{2}$ correspond to the quadrupolar modes, respectively. The efficiencies are related to the crosssections through the following expression: $\mathrm{Q}=\mathrm{C} / \mathrm{G}$, being $\mathrm{G}$ the particle cross-sectional area projected onto a plane perpendicular to the incident beam.

According to the Lorenz-Mie theory, the incident field can be expanded into vector spherical harmonics. Two cases can be considered, one for the electric field polarized perpendicular to the scattering plane $\left(\mathrm{E}_{\mathrm{s}, \text { inc }}\right)$ and another for the electric field lying in the scattering plane, $\left(\mathrm{E}_{\mathrm{p}, \text { inc }}\right)$. The scattered electric-field components are related to the incident ones via the scattering amplitude matrix. For the particular case of linearly polarized light the matrix is diagonal ${ }^{14}$ :

$$
\left(\begin{array}{l}
E_{p S c a} \\
E_{s S c a}
\end{array}\right)=\frac{e^{i \cdot k \cdot(r-z)}}{-i \cdot k \cdot r}\left(\begin{array}{cc}
S_{2} & 0 \\
0 & S_{1}
\end{array}\right)\left(\begin{array}{c}
E_{p I n c} \\
E_{s I n c}
\end{array}\right)
$$


$S_{1}$ and $S_{2}$ are defined from the Lorenz-Mie scattering coefficients $a_{n}$ and $b_{n}$ and the angle-dependent functions $\pi_{n}$ and $\tau_{n}$ as $^{14}$

$$
\begin{aligned}
& S_{1}=\sum_{n} \frac{2 \cdot n+1}{n \cdot(n+1)}\left(a_{n} \cdot \pi_{n}+b_{n} \cdot \tau_{n}\right) \\
& S_{2}=\sum_{n} \frac{2 \cdot n+1}{n \cdot(n+1)}\left(a_{n} \cdot \tau_{n}+b_{n} \cdot \pi_{n}\right)
\end{aligned}
$$

The linear polarization degree of the scattered light in a given direction $\theta$ can be defined as:

$$
P_{L}(\theta)=\frac{I_{s S c a}-I_{p S c a}}{I_{s S c a}+I_{p S c a}}
$$

being $I_{\mathrm{sSca}}$ and $\mathrm{I}_{\mathrm{pSca}}$ the light scattered intensities whose electric field components are perpendicular and parallel to the scattering plane, respectively. $\mathrm{I}_{\mathrm{sSca}}$ and $\mathrm{I}_{\mathrm{pSca}}$ are proportional to $\left|\mathrm{S}_{1}\right|^{2}$ and $\left|\mathrm{S}_{2}\right|^{2}$, respectively.

In particular, at right-angle scattering configuration $\left(\theta=90^{\circ}\right)$, and for a NP size smaller than the incident wavelength, we retain only the first two orders in Eq. (3) ${ }^{8}$ :

$$
P_{L}\left(90^{\mathrm{o}}\right)=\frac{9\left|a_{1}\right|^{2}+25\left|\mathrm{~b}_{2}\right|^{2}+30 \operatorname{Re}\left\{\mathrm{b}_{1} a_{2} *\right\}-9\left|\mathrm{~b}_{1}\right|^{2}-25\left|\mathrm{a}_{2}\right|^{2}-30 \operatorname{Re}\left\{a_{1} b_{2} *\right\}}{9\left|a_{1}\right|^{2}+25\left|\mathrm{~b}_{2}\right|^{2}+30 \operatorname{Re}\left\{\mathrm{b}_{1} a_{2} *\right\}+9\left|\mathrm{~b}_{1}\right|^{2}+25\left|\mathrm{a}_{2}\right|^{2}+30 \operatorname{Re}\left\{a_{1} b_{2} *\right\}}
$$

Indirectly, Eq. (5) may provide information about the order and the electric or magnetic nature of the resonances. It can also be used to determine both the NP size and the refractive index of its surrounding medium. This is because the scattering coefficients are related to the particle size and the relative refractive index. From an experimental point of view, right-angle-detection measurements are simple to execute and were used recently to analyze the spectral behaviour of $\mathrm{P}_{\mathrm{L}}\left(90^{\circ}\right)$ for a single particle made of an HRI material ${ }^{8}$.

\section{RESULTS}

\subsection{Monitoring NP purity through $\mathrm{P}_{\mathrm{L}}\left(\mathbf{9 0}^{\circ}\right)$}

We analyze the effect of the NP purity on the $\mathrm{P}_{\mathrm{L}}\left(90^{\circ}\right)$ spectra. We also prove its use to determine the purity grade of the material. In order to obtain the dielectric constants of contaminant materials, we consider contaminants homogeneously distributed by using a weighted average ${ }^{15}$, in which we have varied the Si concentration from $99.0 \%$ to $100.0 \%$, while the concentration of the main metallic contaminants, considered as $\mathrm{Fe}, \mathrm{Al}$ and $\mathrm{Ti}$, is kept in the initial proportion of most commercial samples $^{16}$. Effective medium theories (EMTs) depend on the geometry and the amount of contaminants ${ }^{17,18}$. Comparisons of EMTs with exact calculations of spherical particles containing inclusions show that although EMTs do not predict the resonance broadening brought on by the scattering of the inclusions, they do predict approximately the resonance shifts due to their presence ${ }^{17}$. We also should note that the studied spheres are much larger than the microscopic NPs of the current study. As the size of the inclusions becomes comparable to the wavelength, these theories tend to lose reliability ${ }^{19}$. Nonetheless, we test the results of these EMTs and find deviations smaller than $5 \%$ for these cases of very small amounts of contaminants. Refractive index values of pure materials are taken from Palik et al. ${ }^{20}$.

As the NP purity decreases, absorption increases and consequently, the extinction resonances weaken. The magnetic resonances are more influenced by the contaminants than the electric ones. 
In order to determine the NP purity by means of the $\mathrm{P}_{\mathrm{L}}\left(90^{\circ}\right)$ measurements we focus on the evolution of the quadrupolar magnetic and dipolar electric and magnetic resonances. We have obtained the value of $\mathrm{P}_{\mathrm{L}}\left(90^{\circ}\right)$ at the wavelengths where the resonances appear, $\lambda_{\text {res}}$, for the following concentrations: $100 \%, 99.88 \%, 99.73 \%, 99.55 \%, 99.37 \%, 99.16 \%$ and $98.98 \%$. The sensitivity of $\mathrm{P}_{\mathrm{L}}\left(90^{\circ}\right)$ on the purity is obtained through the following parameter: $\mathrm{S}_{\mathrm{P}}\left(90^{\circ}\right)$.

$$
S_{P}\left(90^{\circ}\right)=\left|\left(\frac{\partial P_{L}\left(90^{\circ}\right)}{\partial P \operatorname{urity}(\%)}\right)_{\lambda_{\text {res }}}\right|
$$

In Table 1. we show the sensitivity of $\mathrm{P}_{\mathrm{L}}\left(90^{\circ}\right)$ to NP purity for a Si spherical NP of radius $200 \mathrm{~nm}$ embedded in different surrounding media. Concretely, $\mathrm{m}_{\text {med }}$ has been varied from 1.0 to 1.5 with steps of 0.1 .

Table 1. Sensitivity of $\mathrm{P}_{\mathrm{L}}\left(90^{\circ}\right)$ to NP purity where the percent Si ranges from $100 \%$ to $98.98 \%$ for different surrounding media, whose refractive indices range from $\mathrm{m}_{\mathrm{med}} \in[1,1.5]$.

\begin{tabular}{|l|l|l|l|}
\hline \multicolumn{4}{|c|}{$\mathbf{S}_{\mathbf{P}}\left(\mathbf{9 0}^{\mathbf{}}\right)$} \\
\hline $\mathbf{m}_{\text {med }}$ & Quadrupolar Magnetic & Dipolar Magnetic & Dipolar Electric \\
\hline 1.0 & 0.56 & 0.27 & 0.081 \\
\hline 1.1 & 0.58 & 0.26 & 0.081 \\
\hline 1.2 & 0.54 & 0.24 & 0.081 \\
\hline 1.3 & 0.49 & 0.21 & 0.072 \\
\hline 1.4 & 0.46 & 0.17 & 0.045 \\
\hline 1.5 & 0.43 & 0.14 & 0.009 \\
\hline
\end{tabular}

From the obtained results we observe that as the refractive index of the surrounding medium increases, the sensitivity decreases. The magnetic resonances exhibit the highest sensitivities, reaching values near 0.6 when the NPs are in vacuum.

\subsection{Kerker conditions for different purity grades}

As it was mentioned in the introduction, one of the most interesting properties of HRI NPs is their ability to direct incident light in determined directions under certain illumination conditions. Under certain assumptions of the dielectric permittivity and magnetic permeability, the scattered intensity in forward or backward directions is almost null or null, respectively. In the case of the first Kerker condition (or zero-backward intensity condition) the electric and magnetic dipole moments oscillate in phase. However, for the second Kerker condition (or near-zero forward) these dipole moments oscillate out-of-phase. In terms of $\mathrm{P}_{\mathrm{L}}\left(90^{\circ}\right)$, both conditions are satisfied when $\mathrm{P}_{\mathrm{L}}\left(90^{\circ}\right)=0$. The near ZeroForward condition is shifted to longer wavelengths as the purity decreases. However, the Zero-Backward condition is blue-shifted as purity decreases. These shifts are observed in the $\mathrm{P}_{\mathrm{L}}\left(90^{\circ}\right)$ spectra.

It is possible to calculate the sensitivity of the Kerker conditions in the $\mathrm{P}_{\mathrm{L}}\left(90^{\circ}\right)$ to the purity by means of the following expression: 


$$
S_{P}\left(\lambda_{k}\right)=\left|\left(\frac{\partial \lambda_{k}}{\partial \operatorname{Purity}(\%)}\right)\right|
$$

where $\lambda_{\mathrm{k}}$ is the wavelength where the Kerker conditions appear, $\mathrm{P}_{\mathrm{L}}\left(90^{\circ}\right)=0$.

We have obtained the wavelengths where both Kerker conditions appear in the $\mathrm{P}_{\mathrm{L}}\left(90^{\circ}\right)$ spectrum for a Si NP of radius $200 \mathrm{~nm}$ as a function of the purity grade. Using these data and through Eq. 7, we have calculated the sensitivity of this NP to contaminant presence. This process has been repeated for different refractive index of the surrounding medium, $\mathrm{m}_{\operatorname{med}} \in[1,1.5]$.

Table 2 shows the sensitivity of $\mathrm{P}_{\mathrm{L}}\left(90^{\circ}\right)$ to the NP purity grade for a Si spherical NP of radius $200 \mathrm{~nm}$ embedded in different media, $\mathrm{m}_{\mathrm{med}} \in[1,1.5]$ through the wavelength where both Kerker conditions appear. The purity grade has ranged between $100 \%$ and $98.98 \%$.

Table 2. Sensitivity $\mathrm{S}_{\mathrm{P}}\left(\lambda_{\mathrm{k}}\right)$ to the purity ( $\%$ Si from $100 \%$ to $98.98 \%$ ), for different surrounding medium refractive indices, $\mathrm{m}_{\text {med }} €$ $[1,1.5]$.

\begin{tabular}{|l|l|l|}
\hline \multicolumn{3}{|c|}{$\mathbf{S}_{\mathbf{P}}\left(\lambda_{\mathbf{k}}\right)(\mathbf{n m})$} \\
\hline $\mathbf{m}_{\text {med }}$ & $\mathbf{1}^{\text {st }}$ Kerker condition & $\mathbf{2}^{\text {nd }}$ Kerker condition \\
\hline 1.0 & 18.1 & 12.5 \\
\hline 1.1 & 23.6 & 16.7 \\
\hline 1.2 & 27.8 & 19.4 \\
\hline 1.3 & 33.3 & 20.8 \\
\hline 1.4 & 37.5 & 25.9 \\
\hline 1.5 & 41.7 & 0 \\
\hline
\end{tabular}

From Table 2 we show that the first Kerker condition is more sensitive to the change of purity than the second one, reaching values as high as $40 \mathrm{~nm}$ for the maximum $\mathrm{m}_{\text {med }}$ analyzed, $\mathrm{m}_{\text {med }}=1.5$. For both Kerker conditions, sensitivity increases as $\mathrm{m}_{\text {med }}$ increases.

\section{3 $\mathbf{P}_{\mathrm{L}}\left(\mathbf{9 0}^{\circ}\right)$ sensing to $\mathrm{m}_{\text {med }}$ for different purity grades}

It was demonstrated in a recent research that the linear polarization $\mathrm{P}_{\mathrm{L}}\left(90^{\circ}\right)$ measured at resonance locations can be used as a sensor of the surrounding medium refractive index ${ }^{13}$, being the quadrupolar and dipolar magnetic resonances the most and least sensitive, respectively. In this section, we have analyzed the changes of the $\mathrm{P}_{\mathrm{L}}\left(90^{\circ}\right)$ with the surrounding medium refractive index $\mathrm{m}_{\text {med }}$, which was varied from 1.0 to 1.5 , for different Si purity grades.

As the NP purity decreases, absorption increases and consequently, resonances weaken. Apart from the lack of purity, an increase in the refractive index of the surrounding medium also is a contributing factor that weakens resonances. Both factors can be combined to reduce $\mathrm{P}_{\mathrm{L}}\left(90^{\circ}\right)$ values significantly.

The sensitivity of $\mathrm{P}_{\mathrm{L}}\left(90^{\circ}\right)$ to $\mathrm{m}_{\mathrm{med}}$ can be defined as:

$$
S_{m}\left(90^{\circ}\right)=\left|\left(\frac{\partial P_{L}\left(90^{\circ}\right)}{\partial m_{\text {med }}}\right)_{\lambda_{\text {res }}}\right|
$$

The sensitivity, $\mathrm{S}_{\mathrm{m}}\left(90^{\circ}\right)$, is expressed in $\mathrm{RIU}^{-1}$, where RIU are Refractive Index Units ${ }^{21}$. 
Table 3 shows the sensitivity $\mathrm{S}_{\mathrm{m}}\left(90^{\circ}\right)$ to the surrounding medium refractive index $\left(\mathrm{m}_{\mathrm{med}} \in[1,1.5]\right)$ for several grades of purity ranging from $100 \%$ to $98.98 \%$ for a spherical Si NP of radius $200 \mathrm{~nm}$.

Table 3. Sensitivity $\mathrm{S}_{\mathrm{m}}\left(90^{\circ}\right)$ to the surrounding medium refractive index $\left(\mathrm{m}_{\mathrm{med}} \in[1,1.5]\right)$, for different purity grades, $100 \%$, $99.73 \%, 99.55 \%, 99.37 \%$ and $98.98 \%$.

\begin{tabular}{|l|l|l|l|}
\hline \multicolumn{4}{|c|}{$\mathbf{S}_{\mathbf{m}}\left(\mathbf{9 0}^{\mathbf{}}\right)\left(\mathbf{R I U}^{-1}\right)$} \\
\hline Purity (\%) & Quadrupolar Magnetic & Dipolar Magnetic & Dipolar Electric \\
\hline 100 & 1.74 & 0.81 & 1.51 \\
\hline 99.73 & 1.65 & 0.74 & 1.48 \\
\hline 99.55 & 1.81 & 0.69 & 1.45 \\
\hline 99.37 & 0 & 0.64 & 1.43 \\
\hline 98.98 & 0 & 0.52 & 1.38 \\
\hline
\end{tabular}

For each on of the resonances, the sensitivity keeps almost constant with the NP purity. This observation suggests a relatively weak dependence of $\mathrm{P}_{\mathrm{L}}\left(90^{\circ}\right)$ on the purity and a much stronger dependence on the external medium, suggesting that it can be used to determine changes in the surrounding medium refractive index.

Comparing Tables 1 and 3, it is clear that the quadrupolar magnetic resonance is the most sensitive resonance to monitor changes in the media $\mathrm{m}_{\text {med. }}$. Monitoring NP purity is more complicated. For high levels of purity, this quadrupolar resonance is the most sensitive, but when the purity decreases, the dipolar resonances are more sensitive. The electric dipolar resonance is more sensitive than the magnetic dipolar resonance to monitor the refractive index of the surrounding media, while the magnetic dipolar resonance is more sensitive than the electric dipolar resonance to monitor NP purity. The different relative sensitivities of these resonances make it possible to discern changes in the media and NP refractive index.

\section{CONCLUSION}

In this work, we have analyzed the response of the linear polarization degree at right angle scattering configuration for using as a tool to quantify contamination in NPs suspended within different media. Lorenz-Mie calculations were carried out for spherical Si NPs of radius $200 \mathrm{~nm}$. The Si content of the NPs was varied from $100 \%$ to $98.98 \%$ by adding metallic contaminants. We have also considered different values of the refractive index of the surrounding medium

where the particles are embedded, $\mathrm{m}_{\mathrm{med}} \in[1,1.5]$. We have observed that the magnetic resonances are more sensitive to changes in the NP purity than the electric ones, being the quadrupolar magnetic resonance the most sensitive. The sensitivity $\mathrm{S}_{\mathrm{P}}\left(90^{\circ}\right)$ decreases with increasing $\mathrm{m}_{\text {med }}$.

We have also demonstrated another way to detect contamination through shifts observed in the Kerker conditions by means of $\mathrm{P}_{\mathrm{L}}\left(90^{\circ}\right)$. The near Zero-Forward condition goes to longer wavelengths while, the Zero-Backward condition is blue-shifted when the purity grade decreases. The first Kerker condition is more sensitive to NP purity, increasing its value with the increase of $\mathrm{m}_{\text {med. }}$.

Finally, we find that the sensitivity of $\mathrm{P}_{\mathrm{L}}\left(90^{\circ}\right)$ to changes in the external medium refractive index $\mathrm{m}_{\text {med }}$ remains almost constant when the purity of the NP is changed, and that the dipolar electric resonance is more sensitive than the dipolar magnetic resonance to changes of the external medium. This behavior is the opposite of what was obtained for 
contamination. This suggests that by analyzing the sensitivity of all three resonances, it is possible to determine both the level of contamination of the NP and the refractive index of the external medium.

\section{ACKNOWLEDGMENTS}

This research was supported by MICINN (Spanish Ministry of Science and Innovation, project FIS2013-45854-P). Ángela I. Barreda want to express their gratitude to the University of Cantabria for her FPU grant.

\section{REFERENCES}

[1] Prasad, P. N., [Nanophotonics], John Wiley \& Sons, Inc., (2004).

[2] Kelly, K. L., Coronado, E., Zhao, L. L., and Schatz, G., "The optical properties of metal nanoparticles: the influence of size, shape and dielectric environment," J. Phys. Chem. B 107(3), 668-677 (2003).

[3] Forouhi, A. and Bloomer, I., "Optical properties of crystalline semiconductors and dielectrics," Phys. Rev. B 38(3), 1865-1874 (1988).

[4] García-Etxarri, A., Gómez-Medina, R., Froufe-Perez, L. S., López, C., Chantada, L., Scheffold, F., Aizpurua, J., Nieto-Vesperinas, M., and Sáenz, J. J., "Strong magnetic response of submicron silicon particles in the infrared," Opt. Express 19(6), 4815-4826 (2011).

[5] Fu, Y. H., Kuznetsov A. I., Miroshnichenko A. E., Yu, Y. F., and Luk'yanchuk B., "Directional visible light scattering by silicon nanoparticles," Nat. Commun. 4(1527), (2013).

[6] Shi, L., Harris, J. T., Fenollosa, R., Rodriguez, I., Lu, X., Korgel, B. A., and Meseguer, F., "Monodisperse silicon nanocavities and photonic crystals with magnetic response in the optical region," Nat. Commun. 4(1904), (2013).

[7] Gómez-Medina, R., García-Cámara, B., Suárez-Lacalle, I., González, F., Moreno, F., Nieto-Vesperinas, M., and Sáenz, J. J, "Electric and magnetic dipolar response of germanium nanospheres: interference effects, scattering anisotropy, and optical forces," J. Nanophoton. 5(1), 053512 (2011).

[8] Geffrin, J. M., García-Cámara, B., Gómez-Medina, R., Albella, P., Froufe-Pérez, L., Eyraud, C., Litman, A., Vaillon, R., González, F., Nieto-Vesperinas, M., Sáenz, J. J., and Moreno, F., "Magnetic and electric coherence in forward- and back-scattered electromagnetic waves by a single dielectric subwavelength sphere," Nat. Commun. 3(1171), (2012).

[9] García-Cámara, B., Gómez-Medina, R., Sáenz, J. J., and Sepúlveda, B., "Sensing with magnetic dipolar resonances in semiconductor nanospheres," Opt. Express 21(20), 23007-23020 (2013).

[10] Sanz, J. M., Alcaraz de la Osa, R., Barreda, A. I., Saiz, J. M., González, F., and Moreno, F., "Influence of pollutants in the magneto-dielectric response of silicon nanoparticles," Opt. Lett. 39(11), 3142-3144 (2014).

[11] García-Cámara, B., González, F., and Moreno, F., "Linear polarization degree for detecting magnetic properties of small particles," Opt. Lett. 35(23), 4084-4086 (2010).

[12] Setién, B., Albella, P., Saiz, J. M., González, F., and Moreno, F., "Spectral behavior of the linear polarization degree at right-angle scattering configuration for nanoparticle systems," New J. Phys. 12(10), 103031 (2010).

[13] Barreda A. I., Sanz J. M. and González F., "Using linear polarization for sensing and sizing dielectric nanoparticles," Opt. Express 23(7), 9157-9166 (2015).

[14]Bohren, C. F. and Huffman, D. R., [Absorption and Scattering of Light by Small Particles], John Wiley \& Sons, Inc., (1983).

[15]Lesins, G., Chylek, P. and Lohmann, U., "A study of internal and external mixing scenarios and its effect on aerosol optical properties and direct radiative forcing,". J. Geophys. Res. 107(D10), 2002.

[16] Yuge, N., Abe, M., Hanazawa, K., Baba, H., Nakamura, N., Kato, Y., et al., "Purification of metallurgical-grade silicon up to solar grade". Prog. Photovolta. Res. Appl., 9(3), 203-209 (2001).

[17] Mishchenko, M. I., Liu, L. and Mackowski, D. W., "Morphology-dependent resonances of spherical droplets with numerous microscopic inclusions," Opt. Lett. 39(6), 1701-1704 (2014).

[18] Alcaraz de la Osa, R., Moreno, F. and Saiz, J. M., "A new approach for modeling composite materials," Opt. Commun. 291(15), 405-411 (2013). 
[19] Videen, G. and Chylek, P., "Scattering by a composite sphere with an absorbing inclusion and effective medium approximations," Opt. Commun. 158(1), (1998).

[20] Palik, E. D., [Handbook of optical constants of solids], Academic Press, (1998).

[21] Xia, Y. and Hallas, N. J., "Shape-controlled synthesis and surface plasmonic properties of metallic nanostructures," MRS Bull. 30 (5), 338-348 (2005). 